\title{
Twin Boundary Interactions in FCC Metals Observed Using the In-situ TEM Deformation Technique
}

\author{
B.D. Miller and I.M. Robertson
}

Department of Materials Science and Engineering, University of Illinois at Urbana-Champaign, 1304 W. Green St. Urbana, IL 61801

Recently, FCC metals containing large densities of annealing and growth twins have been observed to increase yield strength in engineered grain boundary materials [1] and pulsed electrodeposited copper [2] respectively. To predict the mechanical properties of these materials, dislocation interactions with twin boundaries as well as twin-twin interactions will need to be included in plasticity models. To achieve this aim, these interactions must be identified and the dominating relationships determined. The work described in this paper is aimed at determining this information in a low stacking-fault energy FCC material by using the in-situ TEM deformation technique.

Previous studies have shown that dislocations [3, 4] and stacking faults [5] result from the interaction of glissile dislocations and a twin boundary. For twin-twin interactions, where a growing twin impinges on an existing twin boundary, transmission has been observed to occur by secondary twinning [3], slip [6,7], or both [8]. Recent computer models of nano-grained materials show that the interactions are more complex than previously suggested and that deformation twinning plays an important role in stress transmission at twin boundaries in high SFE materials [9]. These results await experimental verification.

Using the in-situ TEM deformation technique, dislocation interactions with twin boundaries have been observed in a $\mathrm{Cu}-4 \mathrm{wt} \% \mathrm{Al}$ alloy at room temperature. These studies have shown the ejection of perfect dislocations and partial dislocations trailing a ribbon of stacking fault from the same area of grain boundary, the evolution of the dislocation structure including the annihilation of stacking faults, and that deformation twins can act as barriers for perfect and partial dislocations. It was noted that the propagation rate of partial dislocation with the attending stacking fault ribbon and deformation twins was extremely rapid in comparison to the propagation of perfect dislocations. Structures involving partial dislocations would appear within one videoframe, indicating propagation rates faster than 0.03 seconds.

A portion of one interaction in which a stacking fault ribbon is ejected from what appears to be a source near the twin boundary is shown in Figure 1. The fault initially exhibits a lenticular twin shape having an irregular interface with the matrix (Figure 1a). With time, this irregular interface reaches the foil surface along the entire length, creating a uniformly thick twin. At a later time, this fault is released and rapidly moves $\left(1 / 30^{\text {th }} \mathrm{sec}\right)$ out of the frame of reference. The other interactions will be described in more detail elsewhere [10].

\section{References}

1. Blobaum, K.J.M., J.S. Stolken, and M. Kumar. Materials Research Society Symposium Proceedings, v 819, Interfacial Engineering for Optical Properties III, 2004, p 51-56.

2. Lu, L., et al., Science. 2004. p. 422-26. 
3. Mahajan, S. and G.Y. Chin, Acta Metallurgica, 1973. 21: p. 173-79.

4. Remy, L., Acta Metallurgica, 1977. 25: p. 711-14.

5. Malis, T., D.J. Lloyd, and K. Tangri, Phil Mag, 1972. 26: p. 1081-87.

6. Remy, L., Metallurgical Transactions A, 1981. 12A: p. 387-408.

7. Mahajan, S., D.E. Barry, and B.L. Eyre, Phil Mag, 1970. 21: p. 43-52.

8. Radetic, T., V. Radmilovic, and W.A. Soffa, Scripta Materialia, 1999. 40(7): p. 845-52.

9. Yamakov, V., et al., Nature Materials. 2002. p. 45-49.

10. Miller, B.D. and I.M. Robertson, unpublished work.

11. This work was funded by the DOE/SSAAP program under grant DEFG03-02-NA00072. This work was carried out in the Center for Microanalysis of Materials, University of Illinois, which is partially supported by the U.S. Department of Energy under grant DEFG02-91-ER45439
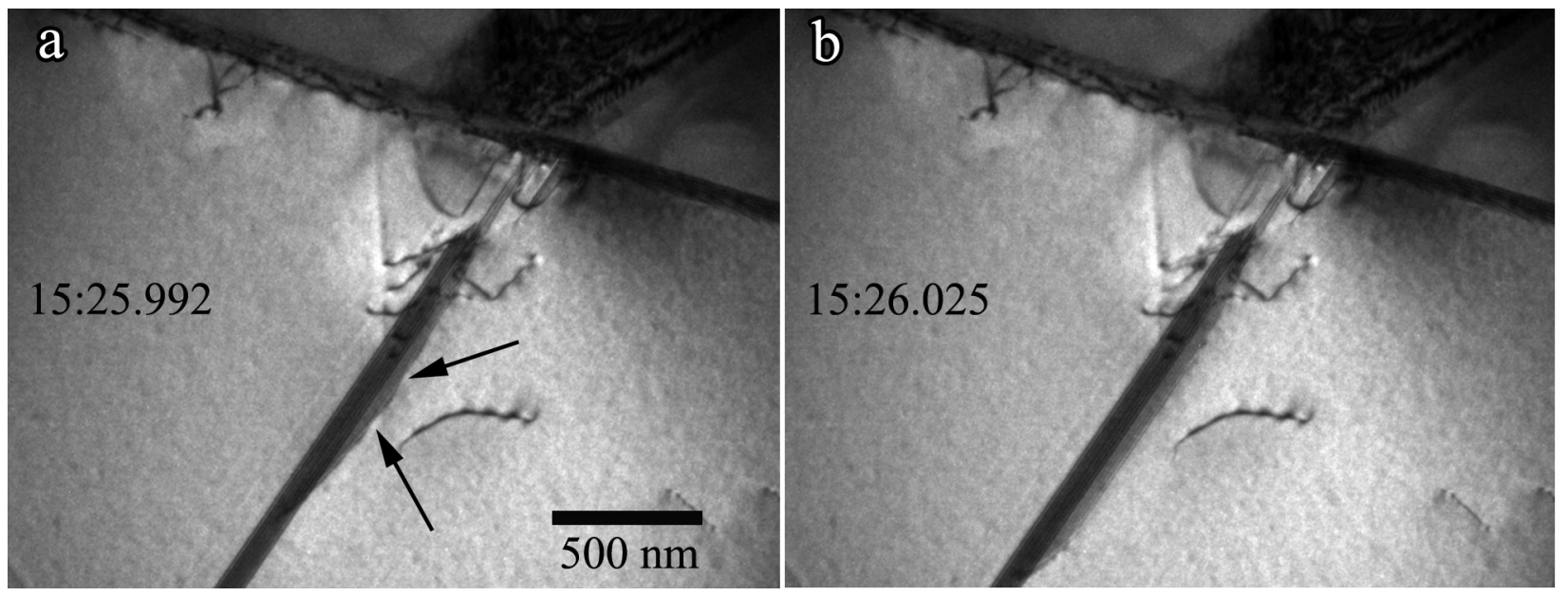

Figure 1. Irregular twin matrix interface that develops during the emission of a stacking fault from a twin boundary in response to a dislocation pileup. Times are shown in minutes:seconds. In Figure 1a, the irregular interface between twin and matrix, denoted by the black arrows, develops on a fault that was initially lenticular in shape. At a rapid rate, $\sim 1 / 30^{\text {th }} \mathrm{sec}$, the interface resumes its lenticular shape as the partial dislocation advances in response to increasing stress from the dislocation pileup. 